\title{
STRUCTURAL STUDIES OF COMPLEX CARBOHYDRATES OF PLANT CELL WALLS
}

\author{
Final Report for the Period \\ December 15, 1989 - June 14, 1993
}

\author{
Dr. Alan G. Darvill \\ Principal Investigator \\ Complex Carbohydrate Research Center \\ The University of Georgia \\ Athens, GA 30602-4712
}

\section{October 1994}

\section{DISCLAIMER}

This report was prepared as an account of work sponsored by an agency of the United States Government. Neither the United States Government nor any agency thereof, nor any of their employees, makes any warranty, express or implied, or assumes any legal liability or responsibility for the accuracy, completeness, or usefulness of any information, apparatus, product, or process disclosed, or represents that its use would not infringe privately owned rights. Reference herein to any specific commercial product, process, or service by trade name, trademark, manufacturer, or otherwise does not necessarily constitute or imply its endorsement, recommendation, or favoring by the United States Government or any agency thereof. The views and opinions of authors expressed herein do not necessarily state or reflect those of the United States Government or any agency thereof.

\section{PREPARED FOR THE \\ U.S. DEPARTMENT OF ENERGY UNDER GRANT DE-FG09-85ER13426}




\section{DISCLAIMER}

Portions of this document may be illegible in electronic image products. Images are produced from the best available original document. 


\title{
STRUCTURAL STUDIES OF COMPLEX CARBOHYDRATES OF PLANT CELL WALLS
}

\author{
Final Report for the Period \\ December 15, 1989 - June 14, 1993
}

Introduction.

Characterizing the polysaccharides that constitute the primary cell walls of plants and developing the methods for these characterizations has been a major research goal of our laboratory for many years. We have made considerable progress in both of these areas during the past three years.

The cell walls of a plant determine the plant's structure and morphology and act as a barrier to pests. Cell walls also control the rate of growth of plant cells and are a source of complex carbohydrates with biological regulatory properties (oligosaccharins). The research projects supported by this grant concentrate on the isolation and structural characterization of the complex carbohydrates that account for approximately $70-80 \%$ of the primary cell walls of dicotyledons and non-graminaceous monocotyledons. We have determined that the pectic polysaccharides (rhamnogalacturonan I, rhamnogalacturonan II, and homogalacturonan) and the hemicellulosic polysaccharides (xyloglucan and arabinoxylan) constitute the predominant non-cellulosic polysaccharides of primary cell walls. Rhamnogalacturonan I includes, among its many covalently attached side chains, arabinans and arabinogalactans, which can be considered distinct polysaccharides.

Over the past three years, our studies focused on the structural characterization of two pectic polysaccharides rhamnogalacturonan I and rhamnogalacturonan II and the hemicellulosic polysaccharide xyloglucan. [In the accompanying proposal to the Department of Energy (The Structures and Functions of Oligosaccharins, \#DE-FG09-85ER13425), we describe our studies of homogalacturonan and its constituent oligogalacturonides.] As described in more detail below, the isolated wall polysaccharides are first chemically and/or enzymatically cleaved to produce oligosaccharides of a size that can be structurally characterized. The structures of these oligosaccharides provide considerable information about the structure of the parent polysaccharide. We have also developed several methods (see below) for the structural characterization of these oligosaccharides that increase both the accuracy and sensitivity of complex carbohydrate structural characterization techniques. Of particular importance is our continuing development of methods using artificial neural network analysis of various forms of spectral information to identify complex carbohydrates. This high-risk, high-payoff research resulted from a request for such an effort by our Department of Energy grants administrator prior to the start of this last three-year funding period. Additionally, we have begun to generate and characterize monoclonal antibodies to specific cell wall epitopes so that we can use the antibodies to localize polysaccharides and polysaccharide substructures within plant cell walls. In these studies we have collaborated with L.A. Staehelin (University of Colorado) and, more recently, have begun collaborations with K. Roberts (John Innes Research Institute, U.K.) and M.S. Fuller (UGA Department of Botany).

The results of our studies on plant cell wall polysaccharides over the last three years confirm our original observations about the tremendous structural complexity of these polysaccharides. However, despite their complexity, we have demonstrated that these non-cellulosic cell wall polysaccharides possess exact, reproducible structures. Moreover, these complex structures are clearly essential to the structure and function of plant cell walls, as we have 
found that they are evolutionarily conserved among plant species. We have only been able to appreciate these structural details because of the availability of sophisticated structural characterization techniques, such as high-field $1 \mathrm{H}$-NMR spectroscopy and several types of mass spectrometry. We have also begun to study the conformations of the oligosaccharide repeating units of xyloglucan, one of the non-cellulosic wall polysaccharides. This is the beginning of our attempts to extend our structural studies of cell wall polysaccharides from primary structural characterization to secondary and even tertiary characterizations. Such information is essential to understand the interactions of the various cell wall polysaccharides, glycoproteins, etc., that form a complete primary cell wall.

Abstracts of papers published, in press, or in preparation reporting work supported by this grant during the past three years follow and summarize more fully the progress made in this research project. [N.B. At the recommendation of the 1989 site visit review committee, this present grant is an amalgamation of two research grants, one on the structure/function of cell walls and the other on methods to determine the structure of complex carbohydrates.]

Publications preceded by an asterisk indicate work also supported by DOE Grant \# DE-FG0985ER13425 (The Structures and Functions of Oligosaccharins); such abstracts are given in that grant's final report.

1. Hahn, M.G., P. Bucheli, F. Cervone, S.H. Doares, R.A. O'Neill, A. Darvill, and P. Albersheim. 1989. Roles of cell wall constituents in plant-pathogen interactions. In: Plant-Microbe Interactions, Vol. 3 (E. Nester and T. Kosuge, eds.) McGraw-Hill, New York, pp. 131-181.

Pathogens find themselves most commonly in the presence of plants other than their hosts. Under such circumstances, a pathogen fails in its efforts to initiate infection. What is it that renders a plant's environment inhospitable to a pathogen except in the rare instance when the plant happens to be a susceptible host? It is this question that we propose to answer by our hypothesis that, in many instances of pathogenesis by bacteria or fungi, it is an interaction between the pathogen and the carbohydrates of the host that determines the pathogen's ability to produce enzymes capable of degrading the host's cell walls.

2. Kiefer, L.L., W.S. York, A.G. Darvill, and P. Albersheim. 1989. Structure of plant cell walls XXVII. Xyloglucan isolated from suspension-cultured sycamore cell walls is O-acetylated. Phytochem. 28:2105-2107.

Xyloglucan oligosaccharides were enzymically released from purified cell walls isolated from suspension-cultured sycamore cells. The O-acetyl substitution pattern of these oligosaccharides was determined by ${ }^{1} \mathrm{H}$ NMR spectroscopy. This analysis revealed that most of the 2linked galactosyl residues of the decasaccharide and nonasaccharide fragments of cell wall xyloglucan are 0 -acetyl substituted, while the heptasaccharide fragment does not contain 0 acetyl substituents. This acetylation pattern does not differ significantly from that of the same xyloglucan oligosaccharides enzymically released from the xyloglucan secreted by suspensioncultured sycamore cells.

* $3 . \quad$ Darvill, A.G., P. Albersheim, P. Bucheli, S. Doares, N. Doubrava, S. Eberhard, D.J. Gollin, M.G. Hahn, V. Marfà-Riera, W.S. York, and D. Mohnen. 1989. Oligosaccharins - plant regulatory molecules. In: Signal Molecules in Plants and PlantMicrobe Interactions (B.J.J. Lugtenberg, ed.) NATO ASI Series, Vol. H36. Springer-Verlag, Heidelberg, pp. 41-48. 
4. Thomsen, J.U. and B. Meyer. 1989. Pattern recognition of the ${ }^{1} \mathbf{H}$ NMR spectra of sugar alditols using a neural network. J. Magn. Res. 84:212-217.

Artificial neural systems (ANS), or neural networks, have produced interesting examples of pattern recognition in several areas of science. To our knowledge, neural networks have not yet been applied in recognition of NMR spectra. We have implemented a software emulation of a three-layered feed-forward neural network as described by Rumelhart et al. to explore the value of ANS in NMR spectroscopy.

5. O'Neill, R.A., P. Albersheim, and A.G. Darvill. 1989. Structure and Function of Plant Cell Walls XXVII. Purification and characterization of a xyloglucan oligosaccharide-specific xylosidase from pea seedlings. J. Biol. Chem. 264(34):2043020437.

An $\alpha$-xylosidase that acts on oligosaccharide fragments of xyloglucan, a plant cell wall polysaccharide, was purified from pea (Pisum sativum) epicotyls that had been treated with an auxin analog. The enzyme had an apparent molecular mass of $85,000 \mathrm{Da}$ according to sodium dodecyl sulfate-polyacrylamide gel electrophoresis and $79,000 \mathrm{Da}$ according to gelpermeation chromatography under nondenaturing conditions. The purified xylosidase consisted of a series of closely related, enzymatically active proteins with isoelectric points ranging from $\mathrm{pH} 7.35$ to 7.7 ; the xylosidases were separated by chromatofocusing. The pH optimum of the mixed xylosidase was 4.9-5.1. The substrate specificity of the xylosidase mixture was determined by purification and structural characterization of the products of treating xyloglucanoligosaccharide substrates with the enzyme. Characterization of the substrates and products included elution volume from a gel-permeation column, glycosyl residue and glycosyl linkage composition analyses, fast atom bombardment-mass spectrometry, and ${ }^{1} \mathrm{H}$ NMR spectroscopy. The enzyme specifically cleaved only one of the $\alpha$-xylosidic linkages of xyloglucan-oligosaccharide substrates, the one attached to a 6-linked glucosyl residue, not those attached to the 4,6 -linked glucosyl residues. The enzyme was unable to cleave the xylosidic linkage of $p$-nitrophenyl- $\alpha$-D-xylopyranoside or the $\alpha$-xylosidic linkage to C-6 of glucose in the disaccharide isoprimeverose. The enzyme was also unable to release measurable amounts of xylose from large xyloglucan polymers.

6. O'Neill, M., P. Albersheim, and A. Darvill. 1990. The pectic polysaccharides of primary cell walls. In: Methods in Plant Biochemistry, Vol. 2 (D.M. Dey, ed.) Academic Press, London, pp. 415-441.

Three - and to date only three - pectic polysaccharides have been isolated from the primary cell walls of plants and characterized. These are homogalacturonan, rhamnogalacturonan I (RG-I), and a substituted galacturonan referred to as rhamnogalacturonan II (RG-II). Homogalacturonan is a chain of 1,4-linked $\alpha$-D-galactosyluronic acid residues in which some (perhaps most) of the carboxyl groups are methyl esterified. RG-I is a family of closely related polysaccharides that contain a backbone of the alternating disaccharide $\rightarrow 4)-\alpha-\mathrm{D}-\mathrm{Gal} p \mathrm{~A}-(1 \rightarrow$ 2)- $\alpha-L-R h a p-(1 \rightarrow$. About $50 \%$ of the rhamnosyl residues are substituted at C-4 with neutral, and perhaps acidic, oligosaccharides. RG-II is a polysaccharide composed of a 1,4-linked $\alpha$-Dgalactosyluronic acid backbone with aldehydo- and keto-sugar oligosaccharide side chains attached to C-2 and/or C-3. Some of the glycosyl residues of the pectic polysaccharides are O-acetylated, but, in general, the location of the acetyl substituents has not been determined. However, RG-I is known to be O-acetylated on C-3 of some of the backbone galactosyluronic acid residues. 
Recent developments in the study of cell-wall pectic polysaccharides, in addition to elucidating new structural features, have emphasized the importance of their biological regulatory functions and physical properties. The heightened interest in structure-function relationships has led to: (1) greater awareness of the problems associated with isolating these complex macromolecules without altering their structures, (2) improved purification procedures for the preparation of homogeneous polysaccharides, and (3) refinements in established analytical procedures and development of new procedures to facilitate the structural characterization of the polysaccharides.

7. Kiefer, L.L., W.S. York, P. Albersheim, and A.G. Darvill. 1990. Structure of Plant Cell Walls XXX. Structural characterization of an arabinose-containing heptadecasaccharide enzymically isolated from sycamore extracellular xyloglucan. Carbohydr. Res. 197:139-158.

An arabinose-containing heptadecasaccharide, generated by endoglucanase digestion of the xyloglucan secreted by suspension-cultured sycamore cells, was structurally characterized. The structure of the heptadecasaccharide was unambiguously determined through combined data generated by ${ }^{1} \mathrm{H}$-n.m.r. spectroscopic, fast-atom bombardment mass spectrometric, and glycosyl-composition and glycosyl-linkage analyses of the intact heptadecasaccharide and of oligosaccharide fragments of the heptadecasaccharide. 1D and 2D ${ }^{1 H}-$ n.m.r. analyses provided data for assignment of the anomeric configurations of the glycosidic linkages, as well as information about the glycosylic and linkage compositions of the heptadecasaccharide. F.a.b.-m.s. data provided the molecular weight and supplied critical information about the glycosylic composition and glycosylic sequence of the heptadecasaccharide. The heptadecasaccharide was found to be a combination of previously characterized nona- and heptasaccharide components of xyloglucan. The nonasaccharide was shown to be glycosidically linked to the heptasaccharide through $\mathrm{C}-4$ of the $\beta$-glucosyl residue nearest to the nonreducing end of the heptasaccharide component. An arabinosyl residue was glycosidically linked at $\mathrm{C}-2$ of the same $\beta$-glucosyl residue at the non-reducing end of the heptasaccharide component of the heptadecasaccharide. Although the presence of arabinosyl residues in sycamore xyloglucan has been recognized since 1973, the location of the arabinosyl residues had not been ascertained.

8. York, W.S., H. van Halbeek, A.G. Darvill, and P. Albersheim. 1990. Structure of Plant Cell Walls XXIX. Structural analysis of Xyloglucan oligosaccharides by ${ }^{1} \mathrm{H}-$ n.m.r. spectroscopy and fast-atom-bombardment mass spectrometry. Carbohydr. Res. 200:9-31.

A method to determine rapidly the identities and proportions of the oligosaccharide repeating-units in plant cell-wall xyloglucans by $1 \mathrm{D}^{1} \mathrm{H}-\mathrm{n} . \mathrm{m} . r$. spectroscopy was developed. Six of the most commonly found xyloglucan oligosaccharide subunits (including three subunits that had not been fully characterized previously) were prepared by endo-(1 $\rightarrow 4)-\beta$-D-glucanase digestion of xyloglucans from various plant species. The oligosaccharides were reduced to the corresponding oligoglycosyl-alditols, purified, and characterized by glycosyl composition and linkage analysis, 1 H-n.m.r. spectroscopy, and f.a.b.-mass spectrometry. Correlations between the $1 \mathrm{H}$-n.m.r. spectra and the structures of the oligoglycosyl-alditols can be used to identify oligoglycosyl-alditols derived from xyloglucans of unknown structure. The identities and relative amounts of the oligosaccharide subunits of xyloglucans isolated from tamarind seed and rapeseed hulls were determined on this basis. 
9. Von Deyn, W., W.S. York., P. Albersheim, and A.G. Darvill. 1990. 1-Alkoxyamino1-deoxy alditols, useful u.v.-absorbing derivatives of neutral and acidic oligosaccharides. Carbohydr. Res. 201:135-144.

The feasibility of using 1-alkoxyamino-1-deoxy alditol derivatives to facilitate the purification and structural characterization of oligosaccharides was examined. 1-Benzyloxyamino-1deoxy, 1-deoxy-1-p-nitrobenzyloxyamino, and 1-deoxy-1-[4-(p-nitrophenyl)butoxyamino] alditol derivatives of mono- and oligosaccharides were prepared by reduction of the corresponding $O$ substituted oximes with sodium cyanoborohydride. The 1-alkoxyamino-1-deoxy alditol derivatives were separated by h.p.l.c. and characterized by ${ }^{1} \mathrm{H}$-n.m.r. spectroscopy and f.a.b.-m.s. Per-O-methylation of the 1-benzyloxyamino-1-deoxy and 1-deoxy-1-p-nitrobenzyloxyamino alditol derivatives resulted in elimination of a benzaldehyde moiety and formation of a quaternary ammonium salt. The positive mode f.a.b.-mass spectra of these cationic per-O-methylated derivatives of oligosaccharides included very intense molecular ion signals, suggesting the possibility of a significant reduction in the amount of per-O-methylated oligosaccharide required for f.a.b.-m.s. analysis. Because the ionic nature of the derivatives hinders their analysis by g.c.-m.s, a new reagent, 4-(p-nitrophenyl)butoxyamine, was synthesized and used to prepare 1-deoxy-1-[4-( $p$-nitrophenyl)butoxyamino] alditol derivatives of several mono- and oligosaccharides. These could be separated by h.p.l.c., with detection by u.v. absorption, and they were stable under $O$-methylation conditions. The per- $O$-methylated derivatives of oligosaccharides can be analyzed by e.i.-m.s. as well as f.a.b.-m.s. O-Methylated 1-deoxy-1-[4-(pnitrophenyl)butoxyamino] alditol derivatives of monosaccharides are amenable to processing by g.c.-m.s., allowing standard methylation analysis of derivatized oligosaccharides to be performed. Thus, the separation and structural analysis of oligosaccharides are facilitated by the physical and chemical properties (u.v. absorption, ease of separation by h.p.l.c., stability to methylation and hydrolysis conditions, and ease of analysis by g.c.-m.s. and f.a.b.-m.s.) imparted to oligosaccharides by forming the 1-deoxy-1-[4-(p-nitrophenyl)butoxyamino] alditol derivatives.

10. York, W.S., L.L. Kiefer, P. Albersheim, and A.G. Darvill. 1990. Oxidation of oligoglycosyl alditols during methylation catalyzed by sodium hydroxide and iodomethane in methyl sulfoxide. Carbohydr. Res. 208:175-182.

Certain artifacts in the glycosyl-linkage analysis of oligoglycosyl alditols were shown to be caused by oxidation of the alditol moieties when O-methylation was catalyzed by $\mathrm{NaOH}$ in $\mathrm{Me}_{2} \mathrm{SO}$. The partially methylated alditol acetates derived from $1-\left[{ }^{2} \mathrm{H}\right]-$ cellobiitol that was $\mathrm{O}$ methylated by this method were analyzed in detail to confirm this conclusion.

11. Sellers, J., W. York, P. Albersheim, A. Darvill, and B. Meyer. 1990. Identification of the mass spectra of partially methylated alditol acetates by artificial neural networks. Carbohydr. Res. 207:C1-C5.

Partially methylated alditol acetates (PMAAs) are used as key glycosyl-residue derivatives in the structural characterization of oligo- and polysaccharides. These derivatives are most readily separated by gas-liquid chromatography (g.l.c.) and identified by electron-impact mass spectroscopy (e.i.-m.s.). Artificial neural networks were originally created to model biological neural networks. Continued development of the underlying mathematical processes has produced networks that can be trained to respond to specific inputs by activating the corresponding output neurons. We have used the mass spectra of the PMAAs of xylitol, arabinitol, rhamnitol, and fucitol to determine whether artificial neural networks can identify mass spectra. Previous work in this laboratory has shown that artificial neural networks are capable of recognizing one-dimensional ${ }^{1} \mathrm{H}$-n.m.r. spectra of alditols and of complex carbohydrates. 
We now report the successful application of artificial neural networks to the recognition of the e.i.-mass spectra of PMAAs.

12. Meyer, B., T. Hansen, D. Nute, P. Albersheim, A. Darvill, W. York, and J. Sellers. 1991. Identification of the ${ }^{1} \mathrm{H}-\mathrm{NMR}$ spectra of complex oligosaccharides with artificial neural networks. Science 251:542-544.

We have found that artificial neural networks can be used to identify hydrogen nuclear magnetic resonance ( ${ }^{1} \mathrm{H}-\mathrm{NMR}$ ) spectra of complex oligosaccharides. Feed-forward neural networks with back-propagation of errors can distinguish between spectra of oligosaccharides that differ by only one glycosyl residue in twenty. The artificial neural networks use features of the strongly overlapping region of the spectra (hump region) as well as features of the resolved regions of the spectra (structural reporter groups) to recognize spectra and efficiently recognized $1 \mathrm{H}$-NMR spectra even when the spectra were perturbed by minor variations in their chemical shifts. Identification of spectra by neural network-based pattern recognition techniques required less than 0.1 second. It is anticipated that artificial neural networks can be used to identify the structures of any complex carbohydrate that has been previously characterized and for which a ${ }^{1} \mathrm{H}-\mathrm{NMR}$ spectrum is available.

13. Hisamatsu, M., G. Impallomeni, W.S. York, P. Albersheim, and A.G. Darvill. 1991. Structure of Plant Cell Walls XXXI. A new undecasaccharide subunit of xyloglucans with two $\alpha$-L-fucosyl residues. Carbohydr. Res. 211:117-129.

A new oligosaccharide subunit of xyloglucan was isolated from the $\beta$-(1 $\rightarrow 4)$-endoglucanase digestion products of the xyloglucan in what is referred to as "sycamore extracellular polysaccharides" and found to be an undecasaccharide having two terminal $\alpha$-L-fucopyranosyl residues. The undecasaccharide was structurally characterized by ${ }^{1} \mathrm{H}-n \cdot m . r$. spectroscopy, fast-atom bombardment mass spectrometry (f.a.b.-m.s.), and glycosyl-residue and glycosyllinkage composition analyses. The structure of the undecasaccharide was confirmed by digesting it with a hydrolase that releases $\alpha-D-X y l p(1 \rightarrow 6)-D-G l c$ from the non-reducing end of xyloglucan oligosaccharides.

14. Doares, S.H., P. Albersheim, and A.G. Darvill. 1991. An improved method for the preparation of standards for glycosyl-linkage analysis of complex carbohydrates. Carbohydr. Res. 210:311-317.

Glycosyl-linkage analysis (i.e., methylation analysis) is a widely used procedure for determining the glycosyl-linkage composition of carbohydrates. Glycosyl linkages are deduced from the electron impact-mass spectra of partially O-methylated, partially O-acetylated alditol derivatives. While the various epimers of a given partially $O$-methylated, partially $O$-acetylated alditol (e.g., 1,3,5-tri-O-acetyl-2,4,6-tri-O-methyl-D-glucitol vs 1,3,5-tri-O-acetyl-2,4,6-tri-O methyl-D-galactitol) give nearly identical mass spectra, they can generally be separated by gas-liquid chromatography (g.l.c.). Thus, complete assignment of the identities of the peaks obtained during g.c.-m.s. of partially O-methylated, partially O-acetylated alditols obtained from an unknown sample relies on the availability of appropriate g.l.c. standards.

Procedures have been developed for the preparation of the required standards for glycosyl-linkage analysis. These protocols involve intentional undermethylation of methyl glycosides, alditols, or aldoses. The mixture of partially methylated aldoses or methyl glycosides is then typically separated from other reactants and products by reversed-phase chromatography on C-18 cartridges or by partitioning into chloroform-methanol. The sample is then 
reduced with sodium borodeuteride (following acid-catalyzed hydrolysis in the case of methyl glycosides) and $O$-acetylated to form a mixture of partially methylated, partially acetylated alditols. These procedures work well for the tetra-O-methyl derivatives of hexoses and for the tri-O-methyl derivatives of deoxyhexoses and pentoses, but these methods produce poor yields of those derivatives carrying fewer $O$-methyl groups. We have found, for example, that the methyl glycosides in a mixture that are only partially O-methylated do not bind efficiently to a reversed-phase cartridge nor do they efficiently extract into chloroform-methanol, due to their more polar nature. Thus, those procedures are strongly biased towards the recovery of partially $O$-methylated, partially $O$-acetylated alditols corresponding to terminal residues, with limited recoveries of the derivatives corresponding to linear glycosyl residues, and essentially no recovery of the derivatives corresponding to branched glycosyl residues.

We describe herein a simple procedure that yields a mixture of all possible partially $O$ methylated, partially $O$-acetylated alditols that can be formed from a given methyl glycoside. In this procedure, a partially methylated methyl glycoside is $O$-acetylated immediately following the undermethylation step to yield a mixture of partially $O$-methylated, partially $O$-acetylated methyl glycosides. The O-acetylation ensures that all components of the mixture are extracted into dichloromethane. The samples are extracted into dichloromethane then hydrolyzed, deuterium-reduced, and re-acetylated. The resulting mixture contains roughly equivalent amounts of each of the possible partially O-methylated, partially O-acetylated alditols theoretically obtainable from the methyl glycoside.

15. O'Neill, M.A., A.G. Darvill, and P. Albersheim. 1991. The degree of esterification and points of substitution by 0 -acetyl and 0 -(3-hydroxybutanoyl) groups in the acidic extracellular polysaccharides secreted by Rhizobium leguminosarum biovars viciae, trifolii, and phaseoli are not related to host range. J. Biol. Chem. 266:9549-9555.

Rhizobium leguminosarum biovars viciae, trifolii, and phaseoli have been grown in the presence and absence of 4',5,7-trihydroxyflavanone (naringenin) or 4',5,7-trihydroxyflavone (apigenin), which induce the expression of nodulation genes of the bacteria. The acidic polysaccharides secreted by the Rhizobium were isolated from the culture media and purified. The polysaccharides were cleaved with a bacteriophage enzyme, and the octasaccharide repeating units formed were isolated. The glycosyl sequence and type of non-glycosyl substituents of the repeating units derived from a number of these Rhizobium biovars were shown by fast atom bombardment-mass spectroscopy and proton nuclear magnetic resonance spectroscopy ('H NMR) to be identical. Minor variations in the degree of esterification of the repeating units by $O$-acetyl and $O$-(3-hydroxybutanoyl) substituents were observed when the Rhizobium were grown in the presence or absence of the flavonoids. The variation in content and the points of attachment of the O-acetyl and O-(3-hydroxybutanoyl) substituents were as great within each Rhizobium biovar as between different Rhizobium biovars and, contrary to two recent reports [Philip-Hollingsworth, S., Hollingsworth, R.I., and Dazzo, F.B. (1989) J. Biol. Chem. 264, 14611466; Philip-Hollingsworth, S., Hollingsworth, R.I., Dazzo, F.B., Djordjevic, M.A., and Rolfe, B.G. (1989) J. Biol. Chem. 264, 5710-5714], the O-acylation patterns were not correlated with the host specificity of the bacteria. 
16. Puvanesarajah, V., A.G. Darvill, and P. Albersheim. 1991. Structural characterization of two oligosaccharide fragments formed by the selective cleavage of rhamnogalacturonan II: Evidence for the anomeric configuration and attachment sites of apiose and 3-deoxy-2-heptulosaric acid. Carbohydr. Res. 218:211-222.

Evidence for the anomeric configurations and attachment sites of 3-deoxy-D-lyxo-2heptulosaric acid (DHA) and apiosyl residues has been obtained through the characterization of two oligoglycosyl fragments isolated from rhamnogalacturonan II (RG-II). One of the oligoglycosyl fragments, a pentaglycosyl aldonic acid generated by Smith degradation of RG-II, was composed of four D-galactopyranosyluronic acid residues, a DHA residue, and a threonic acid residue (derived from a D-galactopyranosyluronic acid residue). The structural analysis of pentaglycosyl aldonic acid established the $\beta$-D-configuration for the DHA residue. Furthermore, it established that a previously identified diglycosyl side chain, 5-O-( $\beta$-L-arabinofuranosyl)-DHA was directly attached to $0-3$ of a D-galactopyranosyluronic acid residue in the backbone of RG-II. The second oligoglycosyl fragment, a peralkylated diglycosyl hex-1-enitol, was generated by hex-5-enose degradation of permethylated and carboxyl-reduced RG-II. The structure of the peralkylated diglycosyl hex-1-enitol, $\beta$-L-Rhap- $\left(1 \rightarrow 3^{\prime}\right)-\beta$-D-Apif-(1 $\left.\rightarrow 5\right)$-hex1-enitol, was determined by a combination of glycosyl-linkage composition analysis and n.m.r. spectroscopy. The n.m.r. data indicated the $\beta$-configuration for the D-apiosyl residue. The isolation and characterization of the diglycosyl hex-1-enitol also established that a previously identified heptaglycosyl side chain was directly attached to 0-2 of a D-galactopyranosyluronic acid in the backbone of RG-II.

17. Koller, A., M.A. O'Neill, A.G. Darvill, and P. Albersheim. 1991. Structure of Plant Cell Walls XXXIII. A comparison of the polysaccharides extracted from dried and non-dried walls of suspension-cultured sycamore cells. Phytochem. 30:39033908.

Suspension-cultured sycamore cells (Acer pseudoplatanus) were disrupted in aqueous $\mathrm{K}$-Pi buffer, and the insoluble residue (the cell wall) purified by extraction with organic solvents and air-dried (dry cell walls) or by washing with aqueous sodium dodecyl sulfate and stored frozen (wet cell walls). Polysaccharides solubilized from the purified wet and dry cell walls by enzymatic digestion and chemical extraction were isolated, and their glycosyl-residue compositions compared. No significant differences were found in the types or yields of the polysaccharides solubilized by enzymatic digestion and chemical extraction of the wet and dry cell wall preparations. Moreover, the glycosyl-residue compositions of the so-called " $\alpha$-cellulose" fraction that remains after extraction of the wet and dry cell wall preparations with alkali was indistinguishable from the glycosyl-residue compositions of the walls prior to extraction.

18. Levy, S., W.S. York, R. Stuike-Prill, B. Meyer, and L.A. Staehelin. 1991. Simulations of the static and dynamic molecular conformations of xyloglucan. The role of the fucosylated sidechain in surface-specific sidechain folding. The Plant $J$. 1:195-215.

The hemicellulosic polysaccharide xyloglucan binds with a strong affinity to cellulosic cell wall microfibrils, the resulting heterogeneous network constituting up to $50 \%$ of the dry weight of the cell wall in dicotyledonous plants. To elucidate the molecular details of this interaction, we have performed theoretical potential energy calculations of the static and dynamic equilibrium conformations of xyloglucan using the GEGOP software. In particular, we have evaluated the preferred sidechain conformations of hexa-, octa-, deca- and heptadecasaccharide model fragments of xyloglucan for molecules with a cellulose-like, flat, glucan backbone 
and a cellobiose-like, twisted, glucan backbone conformation. For the flat backbone conformation, the determination of static equilibrium molecular conformations revealed a tendency for sidechains to fold onto one surface of the backbone, defined here as the $\mathrm{H}_{1}$ s face, in the fucosylated region of the polymer. This folding produces a molecule that is sterically accessible on the opposite face of the backbone, the $\mathrm{H}_{4}$ s face. Typically, this folding onto the $\mathrm{H}_{1} \mathrm{~s}$ surface is significantly stabilized by favorable interactions between the fucosylated, trisaccharide sidechain and the backbone, with some stabilization from adjacent terminal xylosyl sidechains. In contrast, the trisaccharide sidechain folds onto the $\mathrm{H} 4^{\mathrm{S}}$ face of xyloglucan fragments with a twisted backbone conformation. Preliminary NMR data on nonasaccharide fragments isolated from sycamore suspension-cultured cell walls are consistent with the hypothesis that the twisted conformation of xyloglucan represents the solution form of this molecule.

Metropolis Monte Carlo (MMC) simulations were employed to assess sidechain flexibility of the heptadecasaccharide fragments. Simulations performed on the flat, rigid backbone xyloglucan indicate that the trisaccharide sidechain is less mobile than the terminal xylosyl sidechains. MMC calculations on a fully relaxed molecule revealed a positive correlation between a specific trisaccharide sidechain orientation and the "flatness" of the backbone glucosyl residues adjacent to this sidechain. These results suggest that the trisaccharide sidechain may play a role in the formation of nucleation sites that initiate the binding of these regions to cellulose.

Based on these conformational preferences, we suggest the following model for the binding of xyloglucan to cellulose. Nucleation of a binding site is initiated by the fucosylated, trisaccharide sidechain that flattens out an adjacent region of the xyloglucan backbone. Upon contacting a cellulose microfibril, this region spreads by step-wise flattening of successive segments of the backbone. Self-association of xyloglucan molecules in solution may be prevented by the low frequency of formation of these nucleation sites and the geometry of the molecules in solution.

19. O'Neill, M.A., P.D. Robison, K.J. Chou, A.G. Darvill, and P. Albersheim. 1992. Evidence that the acidic polysaccharide secreted by Agrobacterium radiobacter (ATCC 53271) has a seventeen glycosyl-residue repeating unit. Carbohydr. Res. 226:131-154.

The extracellular anionic polysaccharide produced by the bacterium Agrobacterium radiobacter (ATCC 53271) contains D-galactose, D-glucose, and pyruvic acid in the molar ratio 2:15:2. Analysis of the methylated polysaccharide indicated the presence of terminal non-reducing glycosyl, 3-, 4-, 6-, 2,4-, and 4,6-linked glucosyl residues, 3-linked 4,6-O-[(S)-1-carboxyethylidene] glucosyl residues, and 3-linked galactosyl residues. Partial acid hydrolysis of the methylated polysaccharide, followed by reduction with $\mathrm{NaB}^{2} \mathrm{H}_{4}$ and then O-ethylation gave a mixture of alkylated oligoglycosyl alditols that were separated by reversed-phase h.p.l.c. and analyzed by 1 H-n.m.r. spectroscopy, g.l.c.-m.s., and glycosyl-linkage composition analysis. Smith degradation of the polysaccharide gave three diglycosyl alditols that were separated by semi-preparative, high-pH anion-exchange chromatography, and were analyzed by ${ }^{1} \mathrm{H}-$-n.m.r. spectroscopy, g.l.c.-m.s., and glycosyl-linkage composition analysis. The polymer obtained by $\mathrm{NaBH}_{4}$ reduction of the periodate-oxidized polysaccharide was methylated, and the noncyclic acetals were hydrolyzed with aq. $90 \%$ formic acid to generate a mixture of partially O-methylated mono- and diglycosyl alditols. The partially O-methylated oligoglycosyl alditols were O-ethylated. The resulting alkylated oligoglycosyl alditols were separated by reversedphase h.p.l.c. and then characterized by ${ }^{1} \mathrm{H}-$-n.m.r. spectroscopy, g.I.c.-m.s., and glycosyl-linkage composition analysis. The results from the studies described here provide strong evi- 
dence that the acidic polysaccharide secreted by $A$. radiobacter (ATCC 53271) has a heptadecasaccharide repeating unit.

20. Hisamatsu, M., W.S. York, A.G. Darvill, and P. Albersheim. 1992. Structure of Plant Cell Walls XXXV. Characterization of seven xyloglucan oligosaccharides containing from seventeen to twenty glycosyl residues. Carbohydr. Res. 227:4571.

The complete primary structures of seven oligosaccharide subunits of the xyloglucan secreted by suspension-cultured Acer pseudoplatanus cells were determined. The oligosaccharides, ranging in size from 17 to 20 glycosyl residues, were generated by treatment of the xyloglucan with an endo- $\beta-(1 \rightarrow 4)$-glucanase. The oligosaccharide components of a fraction obtained by Bio-Gel P-2 chromatography of enzyme-treated xyloglucan were further purified by normal-phase h.p.l.c. and then converted to the corresponding oligoglycosyl alditols by reduction with $\mathrm{NaBH}_{4}$. The oligoglycosyl alditols, after purification to near homogeneity by reversedphase h.p.l.c., were structurally characterized by ${ }^{1}$ H-n.m.r. spectroscopy, fast-atom bombardment mass spectrometry (f.a.b.-m.s.), and analysis of their glycosyl-residue and glycosyllinkage compositions. Novel structural elements of xyloglucans were observed in this study, including $\beta$-D-xylopyranosyl and $\alpha$-L-arabinofuranosyl-(1 $\rightarrow 3)-\beta-D-x y l o p y r a n o s y l$ sidechains. The results also extend our list of correlations between $1 \mathrm{H}-\mathrm{n}$.m.r. resonances and specific structural features of xyloglucans and thus enhance our ability to determine the structures of xyloglucans from various sources.

*21. Darvill, A., C. Augur, C. Bergmann, R.W. Carlson, J.-J. Cheong, S. Eberhard, M.G. Hahn, V.-M. Lo, V. Marfà, B. Meyer, D. Mohnen, M.A. O'Neill, M.D. Spiro, H. van Halbeek, W.S. York, and P. Albersheim. 1992. Oligosaccharins - oligosaccharides that regulate growth, development and defence responses in plants. Glycobiology 2:181-198.

22. Zhang, G.F. and L.A. Staehelin. 1992. Functional compartmentation of the Golgi apparatus of plant cells. Immunocytochemical analysis of high-pressure frozenand freeze-substituted sycamore maple suspension culture cells. Plant Physiol. 99:1070-1083.

(NOTE: This grant provided the monoclonal antibodies to L.A. Staehelin for these studies as part of our collaborative research.)

The Golgi apparatus of plant cells is engaged in both the processing of glycoproteins and the synthesis of complex polysaccharides. To investigate the compartmentalization of these functions within individual Golgi stacks, we have analyzed the ultrastructure and the immunolabeling patterns of high-pressure frozen and freeze-substituted suspension-cultured sycamore maple (Acer pseudoplatanus L.) cells. As a result of the improved structural preservation, three morphological types of Golgi cisternae, designated cis, medial, and trans, as well as the trans Golgi network, could be identified. The number of cis cisternae per Golgi stack was found to be fairly constant at approximately 1 , whereas the number of medial and trans cisternae per stack was variable and accounted for the varying number of cisternae (3-10) among the many Golgi stacks examined. By using a battery of seven antibodies whose specific sugar epitopes on secreted polysaccharides and glycoproteins are known, we have been able to determine in which types of cisternae specific sugars are added to $N$-linked glycans, and to xyloglucan and polygalacturonic acid/rhamnogalacturonan-I, two complex polysaccharides. The findings are as follows. The $\beta-1,4$-linked D-glucosyl backbone of xyloglucan is 
synthesized in trans cisternae, and the terminal fucosyl residues on the trisaccharide side chains of xyloglucan are partly added in the trans cisternae, and partly in the trans Golgi network. In contrast, the polygalacturonic/rhamnogalacturonan-I backbone is assembled in cis and medial cisternae, methylesterification of the carboxyl groups of the galacturonic acid residues in the polygalacturonic acid domains occurs mostly in medial cisternae, and arabinosecontaining side chains of the polygalacturonic acid domains are added to the nascent polygalacturonic acid/rhamnogalacturonan-I molecules in the trans cisternae. Double labeling experiments demonstrate that xyloglucan and polygalacturonic acid/rhamnogalacturonan-I can be synthesized concomitantly within the same Golgi stack. Finally, we show that the xylosyl residue-linked $\beta-1,2$ to the $\beta$-linked mannose of the core of $N$-linked glycans is added in medial cisternae. Taken together, our results indicate that in sycamore maple suspension-cultured cells, different types of Golgi cisternae contain different sets of glycosyl transferases, that the functional organization of the biosynthetic pathways of complex polysaccharides is consistent with these molecules being processed in a cis to trans direction like the $\mathrm{N}$-linked glycans, and that the complex polysaccharide xyloglucan is assembled exclusively in trans Golgi cisternae and the trans Golgi network.

*23. Hahn, M.G., A. Darvill, P. Albersheim, C. Bergmann, J.-J. Cheong, A. Koller, V.-M. Lo. 1992. Preparation and characterization of oligosaccharide elicitors of phytoalexin accumulation. In: Molecular Plant Pathology (Vol. 2): A Practical Approach (S. Gurr, M. McPherson, D.J. Bowles, eds.). IRL Press, pp. 103-147.

24. Lerouge, P., M.A. O'Neill, A.G. Darvill, and P. Albersheim. 1993. The purification of commercially available endo- $\alpha$-L-arabinanase and $\alpha$-L-arabinosidase for use in the structural analysis of pectic polysaccharides. Carbohydr. Res. 243:373-378.

Commercially available glycanases have considerable potential for use in the structural analysis of complex carbohydrates. However, these preparations often contain mixtures of both endo- and exoglycanases that can limit their use in structural studies. For example, we have found that arabinosyl-containing oligosaccharides released by treating rhamnogalacturonan I (RG-I), a plant cell wall pectic polysaccharide, with a commercially available fungal endo$\alpha-1,5-L$-arabinanase, were hydrolyzed by a contaminating $\alpha$-L-arabinofuranosidase. Fungal arabinases have been purified to apparent homogeneity by fast protein liquid chromatography (FPLC) although their use for the fragmentation of plant cell wall polysaccharides was not reported. We now report that both an endoarabinanase and an $\alpha$-L-arabinosidase can be obtained, by FPLC, in a level of purity suitable for the structural analysis of RG-I and arabinans. The activities of the endoarabinanase and arabinosidase were monitored colorimetrically and by using a method based on high-performance anion-exchange chromatography with pulsed amperometric detection (HPAE-PAD).

25. Lerouge, P., M.A. O'Neill, A.G. Darvill, and P. Albersheim. 1993. Structural characterization of endoglycanase-generated oligoglycosyl side chains of rhamnogalacturonan I. Carbohydr. Res. 243:359-371.

Rhamnogalacturonan I (RG-I) has been isolated from the walls of suspension-cultured sycamore cells (Acer pseudoplatanus), and additional structural features of the polysaccharide elucidated has been further structurally characterized. Treatment of RG-I with a purified endo$1,5-\alpha-L$-arabinanase released a series of arabinose-containing oligosaccharides with degrees of polymerization (DPs) between 2 and 20. These oligosaccharides were shown, by glycosyllinkage composition analysis, to contain terminal, 5-, and 3,5-linked Araf residues. These results provide evidence that a branched arabinan is attached to the backbone of RG-I. RG-I 
was freed of $95 \%$ of its arabinosyl residues by treating the polysaccharide with a combination of endo-1,5- $\alpha$-L-arabinanase and $\alpha$-L-arabinosidase. No galacturonic acid was released by these enzymes, which is evidence that the arabinosyl-containing portions of the side chains do not contain galactosyluronic acid. The galactose-containing portions of the side chains of RG-I were not fragmented by an endo-1,4- $\beta$-D-galactanase. However, approximately $85 \%$ of the galactose and small amounts of galacturonic acid were released by digestion of arabinosedepleted RG-I with a combination of endo- and exo- $\beta$-D-galactanases. The galacturonic acid may have been released by small amounts of an exo- $\alpha$-galactosyluronidase contaminating the galactanases. Treatment of RG-I with this mixture of endo- and exoglycanases resulted in a relatively size-homogeneous, almost side chain-free, backbone composed of the O-acetylated

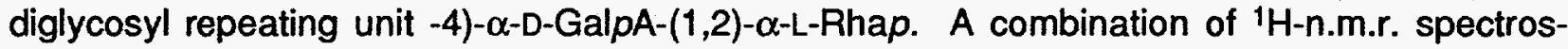
copy and periodate oxidation established that the repeating unit contained a single O-acetyl substituent on $\mathrm{C}-2$ or $\mathrm{C}-3$ of the galactosyluronic acid residue.

26. Spiro, M.D., K.A. Kates, A.L. Koller, M.A. O'Neill, P. Albersheim, and A.G. Darvill. 1993. Purification and characterization of biologically active 1,4 -linked $\alpha-D-o l i-$ gogalacturonides after partial digestion of polygalacturonic acid with endopolygalacturonase. Carbohydr. Res. 247:9-20.

A procedure is described for generating and purifying biologically active $(1 \rightarrow 4)$-linked $\alpha$ D-oligogalacturonides (oligogalacturonides). Oligogalacturonides are generated by treatment of polygalacturonic acid (PGA) with a homogeneous fungal $\alpha-(1 \rightarrow 4)$-endopolygalacturonase (EPG). Oligogalacturonides with a dp greater than seven were selectively precipitated in the presence of sodium acetate and ethanol. Oligogalacturonides with a dp less than eleven and modified oligogalacturonides remained soluble. Oligogalacturonides with dp between 10 and 15 were purified from the resolubilized NaOAc/ethanol-precipitated material using $Q$ Sepharose fast-flow anion-exchange chromatography followed by semipreparative high performance anion-exchange chromatography with pulsed amperometric detection (HPAECPAD). Approximately $70 \mathrm{mg}$ of each homogeneous oligogalacturonide (dp between 10 and 15) was obtained from $10 \mathrm{~g}$ of EPG-treated PGA. The tridecagalacturonide fraction was selected for chemical and structural characterization and was shown to be homogeneous by glycosyl-residue composition analysis, HPAEC-PAD, FABMS, and 1H NMR spectroscopy. . The purified tridecagalacturonide elicited phytoalexin accumulation in soybeans and induced flower formation and inhibited root formation in tobacco thin-cell-layer explants.

27. York, W.S., L.K. Harvey, R. Guillen, P. Albersheim, and A.G. Darvill. 1993. Structural analysis of tamarind seed xyloglucan oligosaccharides using $\beta$ galactosidase digestion and spectroscopic methods. Carbohydr. Res. In press.

The borohydride-reduced forms (oligoglycosyl alditols) of two isomeric octasaccharides $\left(\mathrm{Glc}_{4} \mathrm{Xyl}_{3} \mathrm{Gal}\right)$ that are released from xyloglucans of various plant species upon treatment with a fungal endo- $(1 \rightarrow 4)-\beta$-glucanase were isolated and structurally characterized. A mixture of oligosaccharides that is released from tamarind seed xyloglucan by the endo-(1 $\rightarrow 4)-\beta$ glucanase was digested with a commercially available $\beta$-galactosidase (Aspergillus niger). The $\beta$-galactosidase selectively hydrolyzed the galactosyl residue of one of the two isomeric octasaccharides present in the mixture. A homogeneous preparation of the $\beta$-galactosidaseresistant octasaccharide was prepared by high-resolution gel-permeation chromatography of the enzyme-digestion products. Spectroscopic characterization of the oligoglycosyl alditol prepared by reduction of this octasaccharide confirmed the previously proposed structure that had been based on analysis of the mixture of isomeric octasaccharides. The availability of 
large amounts of the pure, reduced octasaccharide and of a pure, reduced pentasaccharide $\left(\mathrm{Gl}_{3} \mathrm{X} \mathrm{Il}_{2}\right)$ made it possible to completely assign their ${ }^{1} \mathrm{H}$ and ${ }^{13} \mathrm{C}$ NMR spectra. In addition, the borohydride-reduced form of the $\beta$-D-galactosidase-susceptible octasaccharide isomer was purified by high $\mathrm{pH}$ anion-exchange chromatography of the endo- $(1 \rightarrow 4)-\beta$-glucanase-released octasaccharides from rapeseed xyloglucan (no $\beta$-galactosidase treatment), and its $1 H$ and ${ }^{13} \mathrm{C}$ NMR spectra were assigned. Additional correlations between specific structural features of xyloglucan oligoglycosyl alditols and the positions of specific resonances in their NMR spectra were deduced and added to the extensive list that we have compiled. The effects of recording the NMR spectra of the xyloglucan oligoglycosyl alditols in the presence of borate salts, which could lead to incorrect structural assignments, are also described.

28. York, W.S., J.U. Thomsen, and B. Meyer. 1993. The conformations of cyclic $\beta-(1$ $\rightarrow 2$ )-D-glucans: Application of multidimensional clustering analysis to conformational data sets obtained by Metropolis Monte Carlo calculations. Carbohydr. Res. In press.

Sets containing up to $1.3 \times 10^{6}$ energetically accessible conformations of linear $(1 \rightarrow 2)$ $\beta$-D-glucan oligosaccharides were obtained by Metropolis Monte Carlo (MMC) calculations performed with the GEGOP (GEometry of GlycOProteins) program. Quantitative analyses of the data sets (which were expressed in terms of the glycosidic dihedral angle coordinates) were obtained by two different clustering methods: $(I)$ the three-distance hierarchical clustering method (3-DM), published by Jure Zupan, and (ii) a nonhierarchical clustering method (Population-Density Projection, PDP) which, through a segmentation analysis of twodimensional projections of the population-density surface, establishes a partitioning of conformational space into a set of "cluster regions", followed by a clustering step where each conformation of the data set is assigned to one of these regions. Computer programs (MCLUST and PDPCLUST) were developed to perform the 3-DM and PDP analyses, respectively. The two types of analysis provided very similar sets of conformational families (clusters), which could be expressed as combinations of distinct conformations of the glycosidic torsional angles $(\phi, \psi)$ centered at $\left(50^{\circ}, 10^{\circ}\right)$ for conformation $A,\left(40^{\circ}, 160^{\circ}\right)$ for conformation $B,\left(55^{\circ},-160^{\circ}\right)$ for conformation $B^{\prime}$, and $\left(170^{\circ}, 10^{\circ}\right)$ for conformation $C$. The analysis provided the populations of the families, along with relative rates for transitions between families. Examination of the frequencies of the $A, B$, and $C$ glycosidic bond conformations with respect to their relative positions in the sequence revealed the tendency of the $(1 \rightarrow 2)-\beta-D$-glucan to adopt conformational repeating structures of the general form $\left[A_{n} B\right]$, where $n=3$ or 6 . These repeating structures combine in an energetically cooperative fashion to give low-energy cyclic conformations having, for example, $C_{5}$ symmetry $[A A A B]_{5}$ for the eicosamer, and $\mathrm{C}_{3}$ symmetry $\left[\mathrm{AAAAAAB}_{3}\right.$ for the heneicosamer.

29. York, W.S., G. Impallomeni, M. Hisamatsu, P. Albersheim, and A.G. Darvill. 1993. Eleven newly characterized xyloglucan oligoglycosyl alditols: the specific effects of sidechain structure and location on 1H NMR chemical shifts. In preparation.

Eleven previously uncharacterized oligosaccharides, each containing from seventeen to twenty residues, were isolated from the xyloglucan produced by suspension-cultured Acer pseudoplatanus cells and characterized by $1 \mathrm{H}$ NMR spectroscopy, fast atom bombardment mass spectrometry, and matrix-assisted laser-desorption mass spectrometry. The complex mixture of xyloglucan oligosaccharides released by endo-(1 $\rightarrow 4)-\beta$-glucanase (Trichoderma reesel) treatment of cell walls was similar to that released by digestion of the soluble xyloglucan present in the culture medium. The oligosaccharides were converted to 
oligoglycosyl alditols by borohydride reduction and purified by a combination of gel-permeation (Bio-Gel P-2) chromatography, normal-phase HPLC, reversed-phase HPLC, and highperformance anion-exchange (HPAE) chromatography. Eleven new oligoglycosyl alditols (along with several others that had been previously characterized) were isolated and characterized, allowing additional correlations between xyloglucan structure and specific chemical shift effects in the $1 \mathrm{H}$ NMR spectra to be determined. The correlations between structural and spectral features deduced in this study will facilitate the structural determination of a wide range of xyloglucans and their subunit oligosaccharides.

30. Puhimann, J., E. Bucheli, M.J. Swain, N. Dunning, P. Albersheim, A. Darvill, and M.G. Hahn. 1993. Generation of monoclonal antibodies against plant cell-wall polysaccharides. I. Characterization of a monoclonal antibody to a terminal $\alpha-(1 \rightarrow$ 2)-linked fucosyl-containing epitope. In preparation.

Monoclonal antibodies (McAbs) generated against rhamnogalacturonan I (RG-I) purified from suspension-cultured sycamore maple (Acer pseudoplatanus) cells fall into three recognition groups. Four McAbs (group I) recognize an epitope that appears to be immunodominant and is present on RG-I from maize and sycamore maple, pectin and polygalacturonic acid from citrus, gum tragacanth, and membrane glycoproteins from suspension-cultured cells of maize, tobacco, parsley, bean, and sycamore maple. A second set of McAbs (group II) recognizes an epitope present in sycamore maple RG-I but does not bind to any of the other polysaccharides or glycoproteins recognized by group I. Lastly, one $\mathrm{McAb}, \mathrm{CCRC}-\mathrm{M} 1$ (group III), binds to RG-I and more strongly to xyloglucan (XG) from sycamore maple but not to maize RG-I, citrus polygalacturonic acid, or to the plant membrane glycoproteins recognized by group I. The epitope to which CCRC-M1 binds has been examined in detail. Ligand competition assays using a series of oligosaccharides derived from or related to sycamore maple XG demonstrated that a terminal $\alpha-(1 \rightarrow 2)$-linked fucosyl residue constitutes an essential part of the epitope recognized by CCRC-M1. Oligosaccharides containing this structural motif compete with intact sycamore maple $X G$ for binding to the antibody, whereas structurally related oligosaccharides, which do not contain terminal fucosyl residues or in which the terminal fucosyl residue is linked $\alpha-(1 \rightarrow 3)$ to the adjacent glycosyl residue, do not compete for the antibody binding site. The ligand binding assays also indicate that CCRC-M1 binds to a conformationally dependent structure of the polysaccharide. Other results of this study establish that some of the carbohydrate epitopes of the plant extracellular matrix are shared among different macromolecules.

31. Zablackis, E., J. Huang, B. Müller, A.G. Darvill, and P. Albersheim. 1993. Characterization of the cell wall polysaccharides of Arabidopsis thaliana leaves. In preparation.

The cell wall polysaccharides of Arabidopsis thaliana have been isolated, purified and characterized. The primary cell walls of all higher plants that have been studied are composed of cellulose, the three pectic polysaccharides homogalacturonan, RG-1 and RG-II, the two hemicelluloses xyloglucan and glucuronoarabinoxylan, and structural glycoproteins. The cell walls of Arabidopsis leaves contain each of these components and no others that we could detect. The cell walls of Arabidopsis leaves are remarkable in that they are particularly rich in water-soluble polysaccharides (34\%) of the wall). The pectic polysaccharides consist of RG-I $(11 \%)$, RG-II (8\%), and homogalacturonan (23\%). Xyloglucan accounts for $20 \%$ of the wall and the oligosaccharide fragments generated from xyloglucan by endoglucanase consist of the typical subunits of other higher plant xyloglucan. Glucuronoarabinoxylan (4\%), cellulose (14\$) and protein (14\%) account for the remainder of the wall. Except for the water-soluble pectic 
polysaccharides, the polysaccharides of Arabidopsis leaf cell walls occur in proportions similar to those of other plants. The structures of the Arabidopsis cell wall polysaccharides are typical of those of other plants.

32. An, J., M.A. O'Neill, R. Guillen, A.G. Darvill, and P. Albersheim. 1993. Isolation and partial characterization of rhamnogalacturonan I from the stem, leaf, and root of light-grown and etiolated peas. In preparation.

Rhamnogalacturonan I (RG-I), a pectic polysaccharide, has been solubilized from stem, root, and leaf cell walls of light- and dark-grown pea plants by treatment with a fungal endopolygalacturonase. The glycosyl-residue and glycosyl-linkage compositions of RG-I from each type of tissue have been determined. The oligoglycosyl alditols released by lithium fragmentation of each RG-I preparation were compared using a method based on high-performance anion-exchange chromatography with pulsed amperometric detection. The combined results of these studies have shown: (1) RG-I isolated from light-grown pea stems and leaves contains more arabinosyl residues and less galactosyl residues than RG-I isolated from etiolated pea tissue, (2) no major structural differences were detected in RG-I isolated from the roots of both light- and dark-grown plants, (3) the backbone of RG-1 isolated from light-grown tissue contains fewer branched rhamnosyl residues than the backbone of RG-I isolated from etiolated pea tissue, (4) RG-I isolated from etiolated pea tissue contains shorter arabinosyl side chains and longer galactosyl side chains than light-grown RG-I, and (5) RG-I isolated from etiolated pea stem cell walls and root cell walls are structurally similar. These results provide strong evidence that light plays an important role in regulating the structure of a cell wall pectic polysaccharide. 